\title{
Experimental study of wave generation by a granular collapse
}

\author{
Manon Robbe-Saule ${ }^{1}$, Cyprien Morize ${ }^{1, \star}$, Yann Bertho ${ }^{1}$, Alban Sauret ${ }^{1,2}$, and Philippe Gondret ${ }^{1}$ \\ ${ }^{1}$ Laboratoire FAST, Univ. Paris-Sud, CNRS, Université Paris-Saclay, F-91405, Orsay, France \\ ${ }^{2}$ Laboratoire SVI, CNRS, Saint-Gobain, 39 quai Lucien Lefranc, F-93303 Aubervilliers, France
}

\begin{abstract}
We consider experimentally the wave generation by the collapse of a dry granular column into water. Our results show that for a given aspect ratio of the initial granular column, the amplitude of the generated wave depends on the column mass and for a given mass of the granular column, two distinct regimes are observed depending on the aspect ratio of the column $a$ : for $a<3.5$, the volume of the generated wave is directly proportional to $a$ whereas for thinner column, $a>3.5$, there is a saturation of the amplitude of the wave suggesting that the leading wave is generated before the collapse is fully completed.
\end{abstract}

\section{Introduction}

A tsunami is an ocean wave of very large wavelength than can propagate over large distances, typically few thousands of kilometers, and turns into a breaking wave of large amplitude which can cause considerable damages to infrastructures and population when reaching the coasts. Although many tsunamis arise from tectonic events, various past geological events [1] have shown that landslides near coastlines can lead to tsunami waves of significant amplitude. Despite this important hazard, tsunamis generated by landslides remain poorly understood and difficult to model, leading to a very approximated estimate of the associated hazards.

While the generation of tsunamis by a solid block has been widely studied since the 1970s [2], more realistic modeling requires taking into account the granular nature of the landslide. The collapse of dry or immersed granular media has been widely studied during last decades in various situations [3-7]. However, the collapse of dry granular material into water complicates drastically the situation. This complexity mainly arises from the interaction of a two-phases landslide (i.e. the solid beads and the air) into a third phase (i.e. water) and to the inaccurate modeling of the generation of the initial wave. In particular, it is necessary to accurately describe the entry of the granular material into water and the interactions between the grains and the liquid that lead to the generation of the impulse wave [8-10].

In this paper, we consider a model situation consisting in the collapse of a dry granular column into shallow water, leading to the generation of an impulse wave. We focus our attention on the generation mechanisms of the first wave and not on its later propagation.

\footnotetext{
^e-mail: morize@fast.u-psud.fr
}

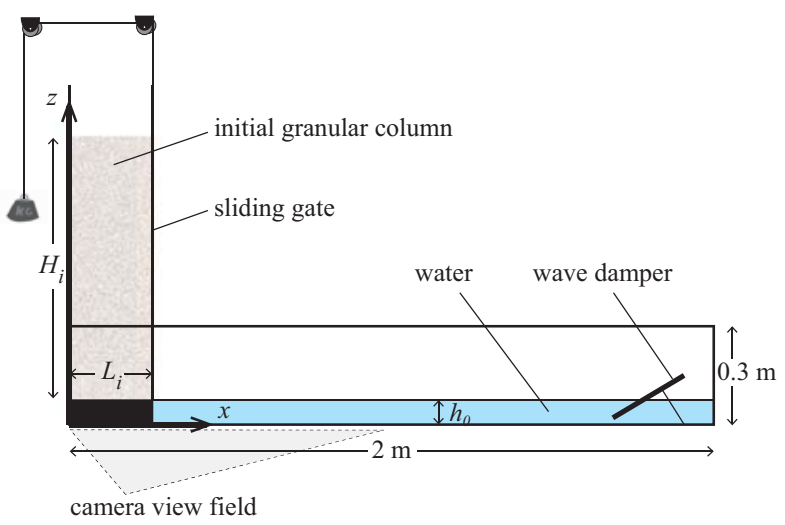

Figure 1. Schematic of the experimental setup.

\section{Experimental setup}

The experimental setup consists of a rectangular tank (2 $\mathrm{m}$ long, $0.3 \mathrm{~m}$ high and $W=0.15 \mathrm{~m}$ wide) in which a sliding gate maintains a column of monodisperse dry grains of height $H_{i}$ and length $L_{i}$, as shown in Fig. 1. The grains are located just above an undisturbed free surface of water of depth $h_{0}=55 \mathrm{~mm}$. Grains are glass beads of density $\rho=2500 \mathrm{~kg} \mathrm{~m}^{-3}$ whose diameter $d$ is $5 \mathrm{~mm}$. This range of diameters ensures that capillary effects can be neglected as the capillary length for water is of the order of $2 \mathrm{~mm}$. In the following, we will note $a=H_{i} / L_{i}$ and $M=\rho \phi H_{i} L_{i} W$, the aspect ratio and the mass of the column respectively, where $\phi \simeq 0.64$ is the packing fraction of the granular column. Upon opening the sliding vertical door, the subaerial granular column collapses with a typical time $\tau^{*}=\sqrt{H_{i} / g}$ and impacts the free surface of water at the typical velocity $u^{*}=\sqrt{g H_{i}}$. This granular collapse generates an impulse wave that propagates at the typical velocity $c=\sqrt{g h_{0}}$ in shallow water conditions. The Froude number is then defined as $F r=u^{*} / c=\sqrt{H_{i} / h_{0}}$. Note that the gate is opened 
fast enough to ensure that it does not affect the dynamics of the granular collapse. The aspect ratio of the tank ensures that the motion of the granular medium and of the generated impulse wave takes place along $x$-direction such that the situation remains two-dimensional. Glass beads of diameter $3 \mathrm{~mm}$ are glued on the bottom of the tank to prevent the grains from sliding over the surface. Finally, an inclined perforated plexiglas plate is located at the end of the tank to absorb the incoming wave and reduce the amplitude of the reflected wave.

The dynamics of the granular collapse and the wave generation are recorded by means of a video camera from the front side of the tank. The processing of the images is realized by using a custom-made Matlab routine based on thresholding method that allows to determine both the surface of the granular collapse and the free-surface $h(x, t)$ of the water during the wave generation. This procedure allows one to extract both the instantaneous amplitude $A(t)=h(x, t)-h_{0}$ and the length $\lambda(t)$ of the impulse wave as the grains collapse, with a resolution of about $0.7 \mathrm{~mm}$. The dynamics of the collapse, the final shape of the deposit and the generated wave are then studied as a function of the aspect ratio of the granular column $a$, the total mass of grains $M$ and their diameter $d$.

\section{Phenomenology}

Figures 2 and 3 show typical examples of a granular collapse of $5 \mathrm{~mm}$ glass beads into water, for two different initial masses $M$ and aspect ratios $a$. Impulse waves, whose dynamics depends on the initial granular column, are observed when the grains impact the free surface of water. Indeed, both the amplitude $A$ and the width $\lambda$ of the generated wave are more important for larger volume of grains, leading to a breaking wave for the largest one (Fig. 3). Note also that in both situations a secondary wave of weaker amplitude is generated after the leading one, but this study goes beyond the scope of this paper.

These two examples highlight the fact that the penetration of a granular column into water is a complex processus involving three different phases: the water, the grains and the air between the grains. Indeed, the wetting of the dry grains is not instantaneous since a front of dry beads clearly appears at short times, below the initial water free surface. This dry/wet front rises progressively during the collapse in a typical timescale of imbibition of an equivalent porous medium. As a result, a volume of air is trapped between the grains in the early stage of the collapse and contributes to displace a given volume of water, and thus partially leads to the wave generation. Finally the air trapped in the granular medium escapes, and bubbles rise to the surface.
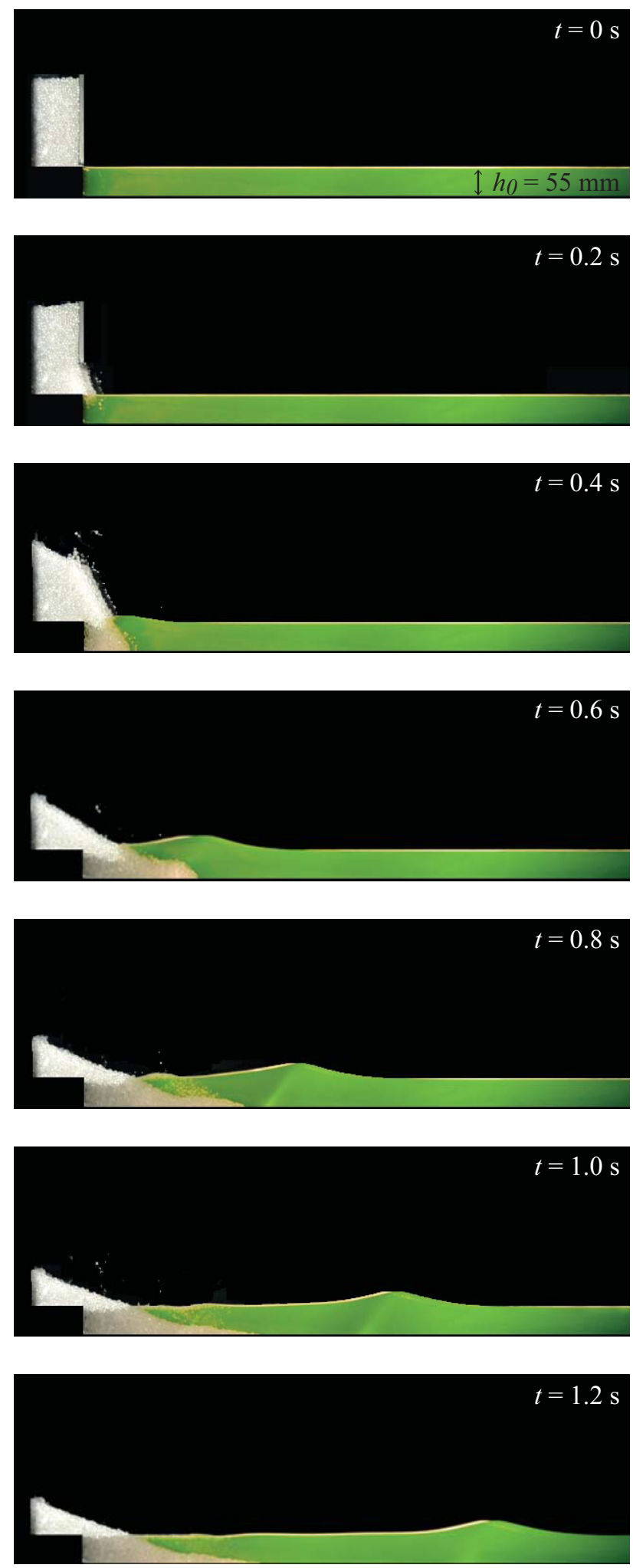

Figure 2. Image sequence showing the propagation of a nonbreaking wave due to the collapse of a granular column (mass $M=2.8 \mathrm{~kg}$, aspect ratio $a=1.8$ ) of $5 \mathrm{~mm}$ glass beads into $h_{0}=55 \mathrm{~mm}$ of water. 

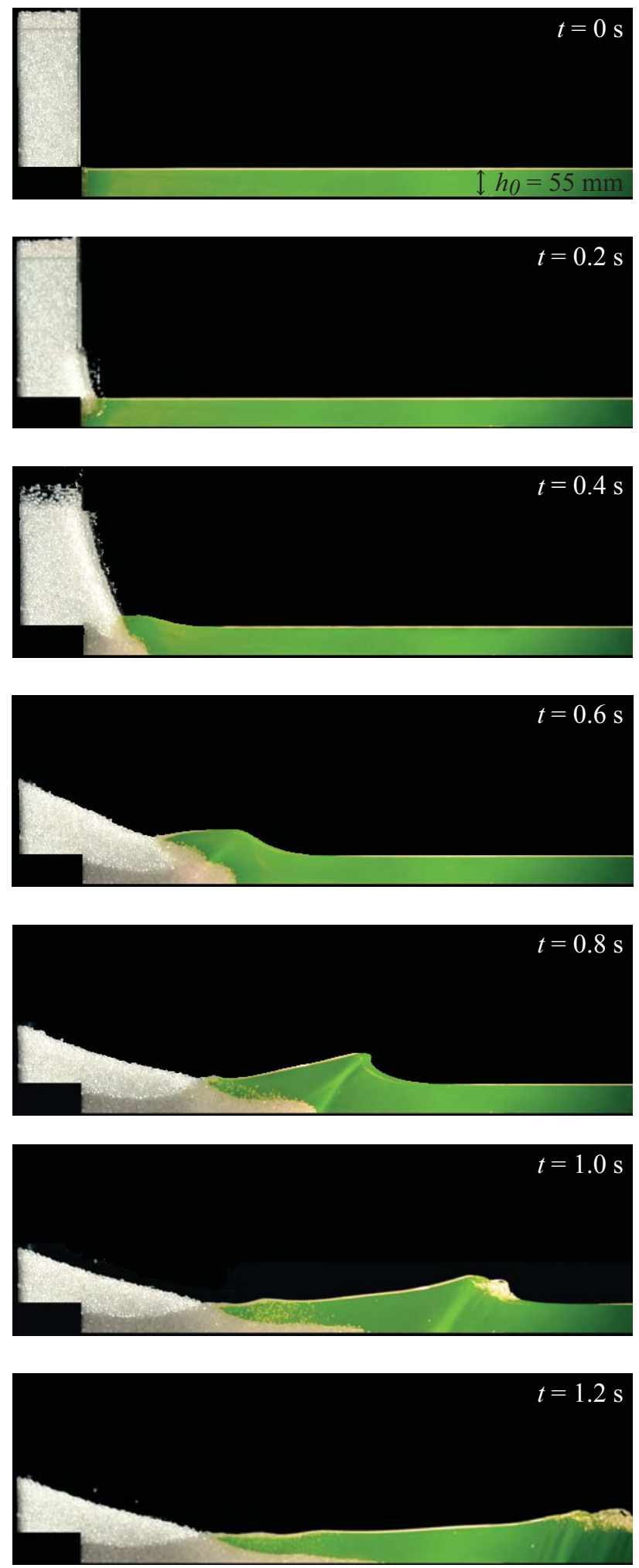

Figure 3. Image sequence showing the propagation of a breaking wave due to the collapse of a granular column (mass $M=6.5 \mathrm{~kg}$, aspect ratio $a=2.7$ ) of $5 \mathrm{~mm}$ glass beads into $h_{0}=55 \mathrm{~mm}$ of water.
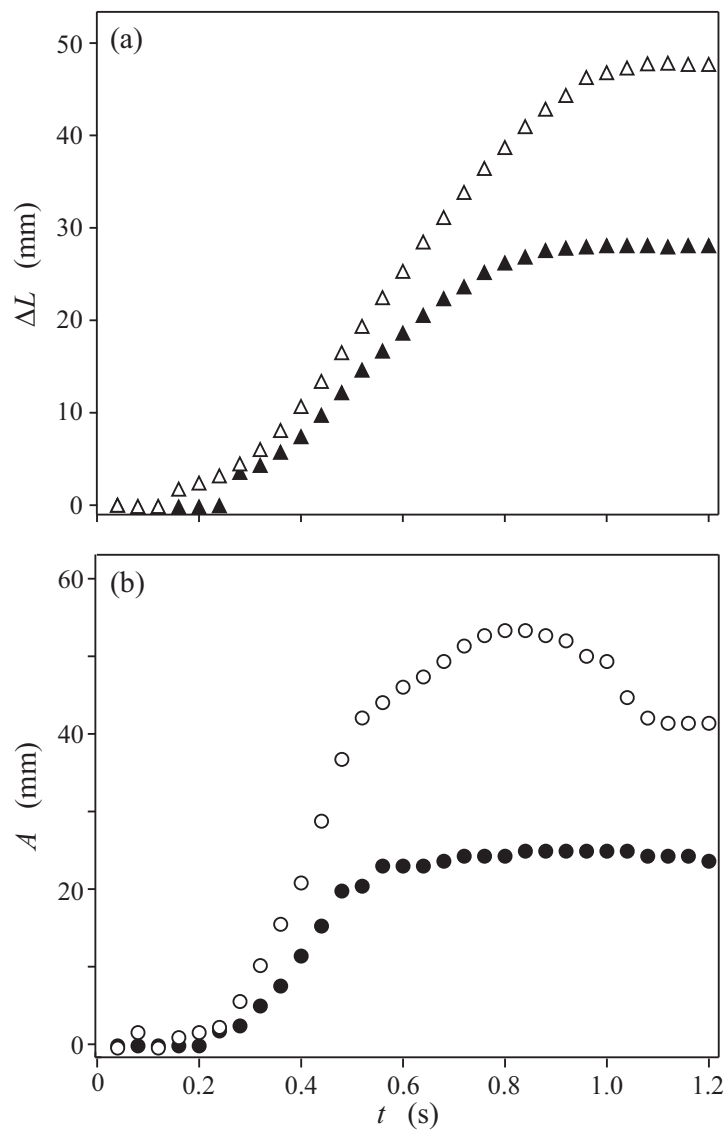

Figure 4. Time evolution of (a) the runout length $\Delta L$ and (b) the amplitude $A$ of leading wave, for the $(\boldsymbol{\Lambda}, \bullet)$ non-breaking and $(\triangle$, $\circ$ ) breaking waves presented in Figs. 2 and 3 respectively.

\section{Experimental results}

The spread of the collapse of the granular column is extracted by measuring the runout distance, in the bottom of the tank at $\mathrm{z}=0$, as a function of time [Fig.4(a)]. Once the collapse of the granular column is achieved, the runout distance is comparable to dry collapses observed in the literature [4].

Figure 4(b) displays the time evolution of the amplitude $A$ of the leading wave generated by the granular collapse for the two experiments presented in Figs. 2 and 3. In both situations, one observes first that this amplitude grows strongly, in a similar way, as the grains collapse into water. Then, for the non-breaking wave, the amplitude reaches a maximum value $A_{\max } \simeq 25 \mathrm{~mm}$. Even if the amplitude of the wave seems to be roughly constant over the small observation window available here, a decay as $x^{-1 / 3}$ is expected, where $x$ is the distance from the point where the wave is generated, in agreement with the weakly dispersive linear shallow water approximation [8]. For larger initial volumes of grains, the amplitude grows until it reaches a larger maximum value $A_{\max } \simeq 54 \mathrm{~mm}$. Once the wave breaks, its amplitude suddenly decreases because of energy dissipation. Thus, the characteristics of the wave generated by a granular collapse highly depend on initial conditions, especially the mass and the initial shape of the granular column impacting the liquid. 


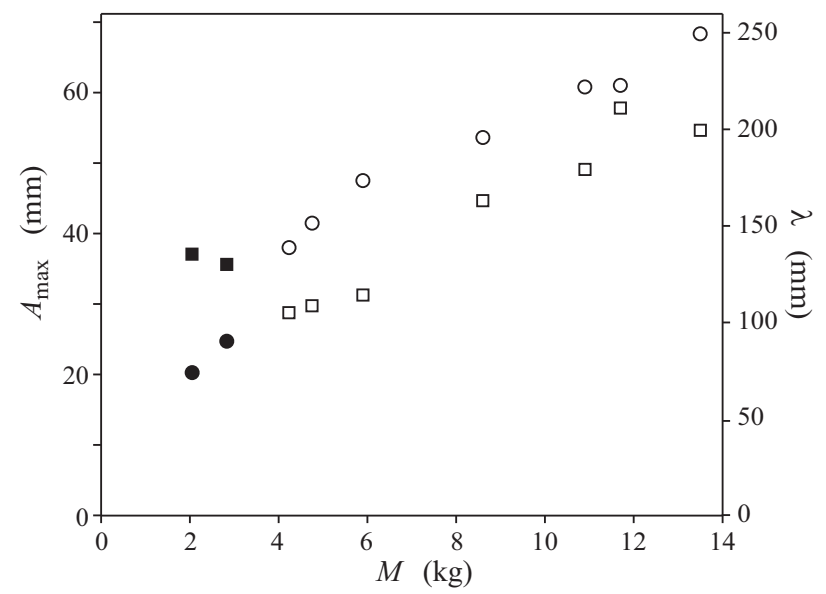

Figure 5. $(\circ, \bullet)$ Maximum amplitude $A_{\max }$ and $(\square, \boldsymbol{\square})$ mid-height width $\lambda$ of the leading wave as a function of the mass of the initial column $M$, for a granular column of aspect ratio $a=2 \pm 0.2$, and of grain diameters $d=5 \mathrm{~mm}$. Empty and filles symbols correspond respectively to breaking and non-breaking waves.

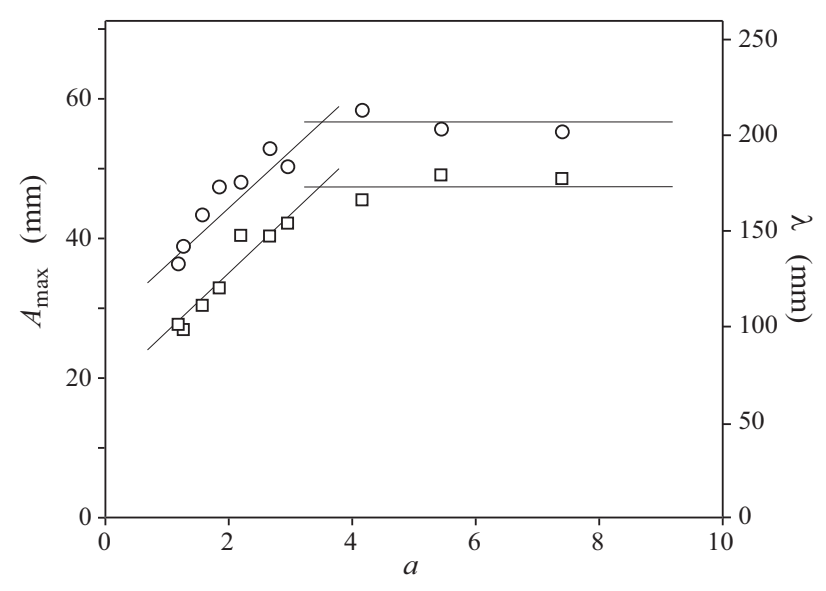

Figure 6. (०) Maximum amplitude $A_{\max }$ and ( $\square$ ) mid-height width $\lambda$ of the leading wave as a function of the aspect ratio $a$ of an initial column of mass $M=6.5 \mathrm{~kg}$ and of grain diameters $d=5 \mathrm{~mm}$. All the data correspond to breaking waves. (-) Guidelines for the eyes.

Figure 5 represents the maximum amplitude $A_{\max }$ and the mid-height width $\lambda$ of the leading wave as a function of the mass $M$ of the initial column, for an initial granular column of aspect ration $a \simeq 2$. Note that $\lambda$ is measured at the mid-height of the leading wave at $t=t_{\max }$ corresponding to the time when the wave amplitude is maximum. Except for the non-breaking waves, one observes that the amplitude and the width of the leading waves increase approximatively linearly with the mass of the granular collapse. Moreover, one can note that the width of the waves is minimum at the transition between non-breaking and breaking waves. This is probably due to the aspect ratio of the breaking waves that have a larger height to width ratio in contrast with non-breaking waves.

Figure 6 represents the maximum amplitude $A_{\max }$ and the mid-height width $\lambda$ of the leading wave as a function of the aspect ratio of the initial granular column $a$, for the same total mass of grains $M=6.5 \mathrm{~kg}$. The amplitude as well as the mid-height width of the generated waves are observed to increase linearly with $a$ for $a \lesssim 3.5$, while they reach a constant value for $a \gtrsim 3.5$. This saturation suggests that for high enough granular columns, the leading wave is generated before the collapse is fully completed. In other words, the grains located at the top of the column are not involved in the wave generation. An estimate of the characteristic times for the generation of the wave and for the granular collapse will help us to understand how the grain column contributes or not to the generated wave.

\section{Conclusion}

By using a small scale laboratory experiment, we investigated, at a very initial state, the generation of waves by the collapse of a dry granular material into water. These experiments show that the amplitude and the width of the generated waves depend on both the initial mass of grains impacting the water surface and the aspect ratio of the granular column. Both the amplitude and the width of the leading waves are found to be proportional to the initial mass of grains for small aspect ratio of the column while they both saturate for larger aspect ratio.

\section{Acknowledgements}

This work is supported by CNRS through its multidisciplinary program "Défi Littoral" via the projects SlideWave and SlideWave2. We are grateful to A. Hildenbrand and S. Viroulet for fruitful discussions. We thank J. Amarni, A. Aubertin, L. Auffray and R. Pidoux for their contribution to the development of the experimental setup.

\section{References}

[1] A. Hildenbrand, P. Y. Gillot, A. Bonneville, Geochem. Geophys. Geosyst. 7 (2006).

[2] P.L.-F. Liu, T.R. Wu, F. Raichlen, C.E. Synolakis, J.C. Borrero, J. Fluid Mech. 536, 107 (2005).

[3] G. Lube, H. E. Huppert, R. S. J. Sparks, M. A. Hallworth. J. Fluid Mech. 508, 175 (2004).

[4] E. Lajeunesse, J.B. Monnier, G.M. Homsy, Phys. Fluids 17, 103302 (2005).

[5] N. J. Balmforth, R. R. Kerswell, J. Fluid Mech. 538, 399 (2005).

[6] L. Rondon, O. Pouliquen, P. Aussillous. Phys. Fluids 23(7), 073301 (2011).

[7] V. Topin, Y. Monerie, F. Perales, F. Radjai, Phys. Rev. Lett. 109, 188001 (2012).

[8] S. Viroulet, A. Sauret, O. Kimmoun, EPL 105, 34004 (2014).

[9] H. M. Fritz, W. H. Hager, H. E. Minor, Exp. Fluids 35, 505 (2003).

[10] M. Cremonesi, A. Frangi, U. Perego, Comput Struct 89, 1086 (2011). 